\title{
Forecasting Macroeconomic Fundamentals in Economic Crises
}

\author{
Maurizio Bovi ${ }^{\dagger} \&$ Roy Cerqueti** \\ ${ }^{\dagger}$ Italian National Institute of Statistics (ISTAT) \\ Piazza dell'Indipendenza, 4, 00185, Rome, Italy \\ Tel.: +3906 46733607; fax: +3906 44482619. Email: mbovi@istat.it \\ $\dagger$ Sapienza University of Rome, Faculty of Economics \\ Via del Castro Laurenziano, 9, 00185, Rome, Italy \\ Email: maurizio.bovi@uniroma1.it \\ $\ddagger$ University of Macerata, Department of Economics and Law \\ Via Crescimbeni 20, 62100, Macerata, Italy. \\ Tel.: +390733 2583246; fax: +390733 2583205. Email: roy.cerqueti@unimc.it
}

\begin{abstract}
The paper studies the way economic turmoils influence the lay agents' predictions of macroeconomic fundamentals. The recent economic crises have, in fact, led several authors to challenge the standard macroeconomic view that all agents are Muth-rational, hence omniscient and homogeneous, forecasters. In this paper lay agents are assumed to be heterogeneous in their predictive ability. Heterogeneity is modeled by assuming that people have equal loss functions, but different asymmetry parameters. The adopted methodological tools are grounded in the standard Operational Research theory. Specifically, we develop a dynamic stochastic optimization problem, which is solved by performing extensive Monte Carlo simulations. Results show that the less sophisticated forecasters in our setting - the medians - never perform as muthians and that second best (SB) agents do that only occasionally. This regardless the size of the crisis. Thus, as in the real world, in our artificial economy heterogeneity is a structural trait. More intriguingly, simulations also show that the medians' behavior tend to be relatively smoother than that of SB agents, and that the difference between them widens in the case very serious crises. In particular, great recessions make SB agents' predictions relatively more biased. An explanation is that dramatic crises extend the available information set (e.g., due to greater mass media coverage), and this leads SB agents, who are more attentive to revise their forecasts than medians. The point is that more information does not necessarily mean better forecasting performances. All considered, thus, our simulations suggest a rewording of Ackoff's famous phrase: it is not silly to not look for an optimal solution to a mess.
\end{abstract}

Keywords: Dynamic stochastic optimization model, economic crisis, forecasting, heterogeneous agents, Monte Carlo simulations.

\section{Introduction}

Expectations of future events play a prominent role in economic decision making. Consumers must think about the type of house to buy, the amount of education to pursue, the

\footnotetext{
${ }^{*}$ Corresponding author.
} 
fraction of income to save, etc. Firms must decide where to locate factories and offices, what products to develop and produce, etc.

Standard macroeconomic theory describes economic decisions as the result of optimizing behavior of omniscient "Muth-rational" agents (Muth, 1961). Muth-rational expectations are a collection of assumptions regarding the manner in which economic agents exploit available information to form their expectations. In its stronger forms, rational expectations operate as a coordination device that permits the construction of a representative agent having representative expectations. Though homogeneous rational expectations are still very commonly assumed in macroeconomics, recently the study of heterogeneous beliefs has been gaining momentum in the literature. This for two main reasons.

First, differences in agents' beliefs play an important role in macroeconomic analysis. For instance, heterogeneity in predictions has been offered as an explanation for why monetary policy shocks can have real and persistent effects on output growth due to $i$ ) limited capacity for processing information (Reis, 2006 and 2009), Mackowiak and Wiederholt, 2009), ii) infrequent updating of beliefs (Mankiw and Reis, 2002) or iii) slow aggregate learning arising from dispersed information (Lorenzoni, 2009). Mixed beliefs may also stem for other reasons. Since the theoretical work of Brock and Hommes (1997), many authors have examined the benefit of including predictor choice as an economic decision in models with expectations formation (Chiarella and Khomin, 1999). Especially in the short run, then, different models may generate different predictions. Nonetheless, by examining GDP dynamics Bovi (2013) finds that, over a time-span of two decades, an easy-to-perform adaptive expectations model systematically outperforms other standard predictors. Though this should reduce model uncertainty and thereby lead to increased homogeneity in expectations, data collected in surveys show that great variety in expectations persists even in this situation. In our setting Bovi's findings are important because they support the notion of the enduring presence of disperse beliefs, in line with the real-world situation. According to Curtin (2008), then, lay people may be interested in knowing about how inflation affects their shopping trolley, or the unemployment rate in their specific labor market, but are less interested in learning about the performance of the whole country or in aggregated macro indicators which are difficult to apply to their daily life. Reis $(2006,2009)$ interprets this kind of finding arguing that costs associated with the acquisition and use of information may generate "rational inattention".

Second, the recent worldwide economic crisis has likely impinged on individuals' predictive ability and, accordingly, on the degree of consensus on expected macroeconomic dynamics - different forecasting performances imply disperse beliefs and vice versa. In this regard, theoretical models (e.g., van Nieuwerburgh and Veldkamp, 2006) suggest that macroeconomic uncertainty and dispersion in beliefs should be greater during recessions. Patton and Timmermann (2010) show supporting evidence of that. Moreover, a better understanding of this kind of heterogeneity can help elaborate sharper tests of macroeconomic models for which subjective beliefs are a driver of economic activity. Though the literature focusing on the prediction of financial crises is flourishing (e.g., Niemira and Saaty, 2004; Sevim et al., 2014), much less effort has been made to examine the impact of bad economic conditions on agents' predictive performance.

In this context, the focus of our work - and intended contribution - is to examine the effects of deteriorated macroeconomic conditions on agents' forecasting performances. The 
fundamental that agents must predict in these economic periods is the GDP growth rate ${ }^{1}$. Specifically, our economic environment is populated by agents featured by different predictive ability caused by disparate attentiveness and/or information sets. Following Capistran and Timmermann (2009), we synthesize this kind of heterogeneity by the asymmetry parameter - namely, $\theta$ - of LINEX loss functions.

We focus on three (groups of) individuals performing reiterate forecasting exercises. The first cluster is made up by "muthians", which are equipped with symmetric -i.e.: $\theta=0$ - LINEX loss functions. They are the benchmark forecasters because they are objectively optimal. The term "objectively optimal" captures the main feature of the muthian agent: it commits (optimal) errors with zero mean (McAllister, 1991). Borrowing from the standard macroeconomic theory, we also hypothesize that there is a bijection between zero-meanerrors agents and muthian ones.

Then, we study "second best" (SB) forecasters, who are able to minimize the distance between their asymmetric loss functions and that of the muthian. The reason for being SB agents can be in the lack of sufficient information and/or the agents' inability to perform at any time as good as the omniscient muthian. SB agents may be though of as expert and/or professional forecasters such as, e.g., econometricians.

Lastly, we examine "median" forecasters. This last cluster is obtained by considering the median of the empirical distribution of the distances between asymmetric and muthian loss functions. Hence, median forecasters can be thought of as agents that structurally follow neither muthian's rationality nor its opposite. Similarly to what said to explain the difference between SB and muthian agents, median and second best forecasters are different because the former have not sufficient skill/information/attention to perform at any time as good as the latter. Medians may be meant as naïve decision makers using rule-of-thumb and/or heuristics (Kahneman, and Tversky, 1974; Bovi, 2009).

We analyze these three (groups of) agents in two alternative bad macroeconomic environments: one only moderately unfavorable, the other much more depressed. The logic is that there is a relationship between the magnitude of the crisis and the agents' forecasting performance. In fact, a well established stylized fact suggests that the more severe the recession, the greater the GDP growth rate volatility. This is not the end of the story, however, because the level of GDP volatility magnifies the level of the forecast error. The basic logic of this latter sequence is easy to understand: a constant is obviously much simpler to forecast than a highly volatile variable (see also Dietz, 2012).

The current crisis may be thought of as a sort of natural experiment to understand how lay decision makers react to very dramatic years. In particular, due to its terrible recent downturn, Greece is one of the most suitable cases, raising the following question: How do Greeks perceive their own personal financial situation with respect to that of their country? Clearly, the representative citizen cannot by definition systematically drift apart from that of the country where she lives, given that the nation-wide economic situation is the (weighted) sum of the individual ones in the country. Yet, it may be hard to remain objective in the course of very deep and prolonged economic crises (Ackoff, 1997).

A unique dataset from the European Commission helps us digging into individuals' perceptions on economic situations. The dataset is based on monthly surveys from the Joint Harmonised EU Programme of Business and Consumer Surveys (European Commission, 2007). These surveys aim at capturing the representative Greek response. Each month

\footnotetext{
${ }^{1}$ Other challenging questions can be addressed by examining some financial variables such as stock prices and returns, volumes, and the like (see e.g.: Chiarella and He, 2001; Consiglio and Russino, 2007). However, our research interest is to explore the expectations formation process on real macroeconomic fundamentals.
} 
respondents answered, among others, the following two questions: "How do you expect the financial position of your household to change over the next 12 months?" and "How do you expect the general economic situation in the country to develop over the next 12 months?" The responses are summarized in two indexes, one for each question, varying between +100 (all respondents answer "It is getting a lot better") and -100 (all respondents answer "It is getting a lot worse").

Figure 1 shows the squared difference between the two indexes since 1985.

\section{INSERT FIGURE 1 ABOUT HERE}

Caption: Squared difference between the indices which summarize the answers of the Greek citizens to the following two questions: "How do you expect the financial position of your household to change over the next 12 months?" and "How do you expect the general economic situation in the country to develop over the next 12 months?".

Responses vary between +100 (all respondents answer "It is getting a lot better") and -100 (all respondents answer "It is getting a lot worse"). The considered period is 1985M1 - 2013M7.

Evidence as clearly as astonishingly shows that, hit by the sovereign debt crisis started in 2008 , month-after-month for nearly two years, there was an unprecedented level of decoupling between the Greeks' predictions of their own and their nation's economic destiny. We interpret this persistent misalignment between Greeks and Greece as supporting evidence that greater political and economic turbulence makes it harder to maintain objectivity. In turn, it may imply that during great recessions $i$ ) decision makers could behave less optimally than in less extreme cyclical phases and that $i i$ ) heterogeneity may become wider. In this paper we try to shed some light on this kind of issues.

Based on the above as well as on the existing evidence (Aizenman and Pinto, 2005), we assume that the level of macroeconomic volatility increases with the gravity of the turmoil. To learn the effects of deteriorated economic conditions on agents' forecasting exercises we develop a stochastic dynamic optimization problem. From the methodological point of view, our paper belongs and intends to add to the field of the optimal control theory. The reader can find a complete survey of deterministic mathematical control theory in Bardi and Capuzzo Dolcetta (1997). The stochastic framework is described in Borkar (1989), Fleming and Soner (1993), Krylov (1980), Yong and Zhou (1999). In general, according to some authors, optimization theory is a particularly useful tool for doing macroeconomic analysis (see e.g. Engels, 1992; Woodford, 2009; Consiglio and Staino, 2012). Modeling of economic crises based on the optimization theory and operations research has already been done by some scholars in a number of different frameworks. To cite only a few: Kirby (2007) deals with the relationships between confidence in operations research and economic turbulence; Bayram et al. (2014) develop a stochastic integer program to detect the strategies of the so-called community development corporations, non-governmental/nonprofit entities contrasting the crisis in the U.S. by investing in foreclosed properties. Rios-Rull (2001) is somewhat closer to our paper in that he deals with the assessment the relationship between business cycle and heterogeneity of beliefs by developing dynamic optimization models. The main aim of the present study, however, is the way poor macroeconomic performances affect lay people's predictions of real GDP dynamics.

From the operative standpoint, we present a constrained minimization problem where the objective function is the distance between the muthian's loss function and the asymmetric one. The problem is solved with respect to time-dependent asymmetry parameters $\theta$ 's (the "control variables"). These latter actually control the objective function and the dynamics 
of the errors (the "state variable", describing the evolution of the system). The control variables belong to a specific functional space (the "admissible region"). The adopted solution strategy for the optimal control problem is to implement extensive Monte Carlo simulations. In doing this, we are in line with a wide strand of the literature (see e.g. Cao et al., 2003; Barone et al., 2012; Cerqueti and Quaranta, 2012; Blueschke et al., 2013, Castellano and Cerqueti, 2013, 2014). By adopting such a solution strategy, two targets are achieved: i) providing a more intuitive discussion of the economic sense of the optimal solution; $i i$ ) maintaining the theoretical model as general as possible, avoiding restrictive mathematical assumptions by making its quantitative treatment possible.

Simulations show intriguing patterns, shedding some light on how the asymmetry parameters - i.e. the agents' predictive ability - vary according to the seriousness of the crisis. First, SB agents can succeed in forecasting as good as muthians. As expected, however, this happens only occasionally and never happens for medians. Therefore, our artificial economy is able to capture the structural heterogeneity characteristic of the real world. The less sophisticated forecasters' $\theta$ 's tend to follow relatively smoother paths than the second best's ones. This holds regardless of the size of the crisis. The detected less-schizoid behavior may mirror the fact that, by definition, medians have less-than-optimal forecasting ability and/or attention and/or lower information than SB individuals. Accordingly, they are less prone or prompt to seek and exploit new information and to significantly revise their beliefs. Moreover, the SB forecaster tend to stay as close as possible to Muth-rationality and turbulence tends to enlarge muthians' forecasting errors. In fact, another remarkable finding of our simulations is that our agents are relatively more similar during mild economic periods, while behaving very differently during periods of economic turmoils. In particular, it seems that major crises lead only SB agents to adopt a sort of bang-bang behavior. An explanation is that very serious crises broaden the information set - e.g., due to greater mass media coverage - possibly leading SB agents to substantially revise their the forecasts, since they are the ones who are more prone to rapidly take into account any new information. The problem with this beliefs reshuffle is that, as emphasized by Doms and Morin (2004), the media tend to emphasize bad economic news. Whilst this pertains to the natural approach of the media to the news, Blendon et al. (1997) have pointed out that the public's biases can be attributed to the media's focus on bad news (see also Bovi, 2009). From this perspective, many years ago Simon (1971) stressed that a wealth of information creates a poverty of attention. Hence, more information neither necessarily mean better information nor better forecasting performances. All in all, thus, our simulations suggest a rewording of Ackoff's famous phrase: it is not silly to not look for an optimal solution to a mess.

The rest of paper is organized as follows: the next Section contains the main ingredients of the model and the formalization of the optimization problem. Section 3 describes the Monte Carlo procedure employed to solve the optimization problem. Section 4 lists and discusses the obtained results. Concluding remarks close the paper. The pseudo-codes of the simulations are reported in the Appendix.

\section{The Model}

We introduce a filtered probability space $\left(\Omega, \mathcal{F},\left\{\mathcal{F}_{t}\right\}_{t \geq 0}, \mathbb{P}\right)$ which contains the random quantities of the model.

The economic environment is populated by a group of heterogeneous agents, who are assumed to operate in a period characterized by a negative cyclical phase. GDP dynamics 
at time $t$ will be denoted as $f_{t}$, while the evolution of agents' beliefs on GDP growth is collected in

$$
\left\{\tilde{\mathbf{f}}_{t}\right\}_{t \geq 0}=\left\{\left(\tilde{f}_{t}^{(i)}\right)_{i=1, \ldots, N}\right\}_{t \in \mathbb{N}} .
$$

Each agent forecasts the rate of change of the GDP and tries to maintain her/his forecasts in line with the actual rate of change in a sense that will be cleared later on. Agents' beliefs evolve deterministically according to a controlled difference equation:

$$
\tilde{\mathbf{f}}_{t}=\tilde{\mathbf{f}}_{t-1}+\beta\left(\tilde{\mathbf{f}}_{t}, \theta_{t}\right),
$$

where:

- the $N$-variate stochastic process

$$
\left\{\theta_{t}\right\}_{t \in \mathbb{N}}=\left\{\left(\theta_{t}^{(i)}\right)_{i=1, \ldots, N}\right\}_{t \in \mathbb{N}}
$$

describes the heterogeneity among agents. For each $i=1, \ldots, N$ and $t \in \mathbb{N}$, the value $\theta_{t}^{(i)}$ represents the asymmetric parameter at time $t$ of the LINEX cost function employed by the agent $i$ to perform predictions (for the definition of the LINEX cost function, see the next section). The functional space $\Theta$ is the admissible region, and it is defined as follows:

$$
\Theta:=\left\{\theta^{(i)}:[0,+\infty) \times \Omega \rightarrow \mathbb{R}\left\{\mathcal{F}_{t}\right\} \text { - adapted processes }\right\} .
$$

- $\beta: \mathbb{R}^{N} \times \mathbb{R}^{N} \rightarrow \mathbb{R}^{N}$, where

$$
\beta\left(\tilde{\mathbf{f}}_{t}, \theta_{t}\right)=\left(\beta_{i}\left(\tilde{f}_{t}^{(i)}, \theta_{t}^{(i)}\right)\right)_{i=1, \ldots, N}
$$

- $\beta_{i}$ is a continuous real value bounded function with respect to the process $\theta_{t}^{(i)}$, for each $i=1, \ldots, N$;

- $\tilde{\mathbf{f}}_{0} \in \mathbb{R}^{N}$ is the deterministic initial value of the dynamics described in (1).

On the basis of standard economic evidence, we assume that the variance of the real GDP is affected by the severity of the occurring economic crisis. To formalize this hypothesis, we introduce the negativity level of the business cycle at time $t$ through an aggregated index $\alpha_{t} \in[-1,0)$, which is assumed to collect all the available information related to the entity of the crisis and to work as follows: fixed $t \in \mathbb{N}$, the lower the value of $\alpha_{t}$, the more severe the negativity level of the business cycle. The specific case of $\alpha_{t} \sim 0^{-}$means that crisis is not so deep, and the economy is stagnant.

GDP dynamics is hypothesized to be driven by a stochastic difference equation:

$$
f_{t}=f_{t-1}+\mu_{f}\left(f_{t}\right)+\sigma_{f}\left(f_{t}, \alpha_{t}\right) \epsilon, \quad t>0,
$$

where:

- $\mu_{f}: \mathbb{R} \rightarrow \mathbb{R}$

- $\sigma_{f}: \mathbb{R} \times[-1,0) \rightarrow[0,+\infty)$; 
- $\epsilon \sim N(0,1)$ is a standard normal random variable.

We assume that the initial value of the rate of change of the GDP, $f_{0}$, is deterministic and, as such, it is known. Moreover, there is widespread evidence that the GDP is more volatile in periods of recession than when the GDP growth is close to zero. Hence, at time $t, \sigma_{f}$ is reasonably assumed to be increasing with respect to $\left|\alpha_{t}\right|$.

We define the state-variable

$$
\left\{\mathbf{x}_{t}\right\}_{t \in \mathbb{N}}=\left\{\left(x_{t}^{(i)}\right)_{i=1, \ldots, N}\right\}_{t \in \mathbb{N}}=\left\{\left(f_{t}-\tilde{f}_{t}^{(i)}\right)_{i=1, \ldots, N}\right\}_{t \in \mathbb{N}}
$$

whose evolution, given (3) and (1) is given by:

$$
\mathbf{x}_{t}-\mathbf{x}_{t-1}=\left(\mu_{i}\left(x_{t}^{(i)}, \theta_{t}^{(i)}\right)+\sigma\left(x_{t}^{(i)}, \alpha_{t}\right) \epsilon\right)_{i=1, \ldots, N}, \quad t>0
$$

where:

- $\mu_{i}\left(x_{t}^{(i)}, \theta_{t}^{(i)}\right)=\mu_{f}\left(f_{t}\right)-\beta_{i}\left(\tilde{f}_{t}^{(i)}, \theta_{t}^{(i)}\right)$;

- $\sigma\left(x_{t}^{(i)}, \alpha_{t}\right)=\sigma_{f}\left(f_{t}, \alpha_{t}\right)$;

- $\mathbf{x}_{0}=\left(x^{(i)}\right)_{i=1, \ldots, N}$ is the deterministic starting point of the dynamics $\left\{\mathbf{x}_{t}\right\}_{t \in \mathbb{N}}$.

For each $i=1, \ldots, N$, functions $\mu_{i}$ and $\sigma_{i}$ and their arguments are assumed to satisfy the usual regularity conditions for the existence and uniqueness of the solution for the $i$-th equation with initial data introduced in (4).

\subsection{The optimization problem}

We assume that agents perform their forecasting exercises simultaneously and that there is no imitative nor strategic behavior. It is worth recalling that the heterogeneity across our agents is due to their different level of skills and/or information and/or attention. Each agent has the same loss function, which is minimized with respect to the errors $x$ 's to perform the forecasting exercise. Specifically, agent $i$ aims at minimizing her/his loss function by appropriately selecting her/his asymmetry parameter.

Following the literature (Capistran and Timmermann, 2009; Pesaran and Weale, 2006) as loss function we use the LINEX (see Varian, 1975). This cost function is widely adopted in the literature dealing with expectations on macroeconomic fundamentals because, in the extreme case, asymmetric loss permits forecasters to be muthian, i.e. fully-informed optimal, forecasters while still accounting for the empirical observation that forecasters "disagree", provided that their loss functions exhibit different degrees of asymmetry. In addition, the LINEX is widely adopted because Capistran and Timmermann (2007) show that one can obtain essentially the same conclusions in the case of a general asymmetric loss function, provided only weak restrictions are put on the form of that loss function and the data-generating process (DGP). The requirement of the DGP is that the variable of interest is conditionally location scale distributed, and the requirement of the loss function is that it is homogeneous in the forecast error. Therefore, the LINEX allows capturing the presence of asymmetry, which is not the case for other functions such as the quadratic and the log. Finally, it is worth noting that the quadratic loss function is a particular case of 
the LINEX with $\theta=0$, as easily seen in the symmetric case of the following eq. (5)). Our LINEX is $L: \mathbb{R}^{2} \rightarrow \mathbb{R}$ such that:

$$
L\left(x_{t}^{(i)}, \theta_{t}^{(i)}\right)= \begin{cases}\mathbb{E}\left\{\frac{1}{\left[\theta_{t}^{(i)}\right]^{2}} \cdot\left[\exp \left(\theta_{t}^{(i)} \cdot x_{t}^{(i)}\right)-\theta_{t}^{(i)} \cdot x_{t}^{(i)}-1\right]\right\}, & \text { for } \theta_{t}^{(i)} \neq 0 \\ \mathbb{E}\left\{\left[x_{t}^{(i)}\right]^{2}\right\}, & \text { for } \theta_{t}^{(i)}=0\end{cases}
$$

where $\mathbb{E}$ represents the usual expected value operator, $\theta_{t}^{(i)}$ is the asymmetric loss parameters at time $t$ of the agent $i$, and its sign describes the asymmetry property of $L$ associated to the $i$-th agent. When $\theta_{t}^{(i)}>0\left(\theta_{t}^{(i)}<0\right)$, then the loss function increases with respect to $x_{t}^{(i)}$ when $x_{t}^{(i)}>0\left(x_{t}^{(i)}<0\right)$ and decreases otherwise. As $\theta_{t}^{(i)}$ approaches 0 , then the LINEX function approaches the symmetric case $L\left(x_{t}^{(i)}, 0\right)=\mathbb{E}\left\{\left[x_{t}^{(i)}\right]^{2}\right\}$.

In our setting, agents are able to change the asymmetry parameter of their loss function at each time $t$.

Remark 1. If $\theta_{t}^{(i)}=0$, then the forecaster will have errors with zero mean. In fact, we have:

$$
\min _{x_{t}^{(i)} \in \mathbb{R}} L\left(x_{t}^{(i)}, 0\right)=\min _{x_{t}^{(i)} \in \mathbb{R}} \mathbb{E}\left\{\left[x_{t}^{(i)}\right]^{2}\right\}=0,
$$

if and only if $\mathbb{E}\left\{x_{t}^{(i)}\right\}=0$.

In this case, we refer to the muthian agent at time $t$. Muthian agent has a complete informative set and full ability to exploit such information.

Of course, when the dynamics of the errors in (4) is such that $\mathbb{E}\left\{x_{t}^{(i)}\right\} \neq 0$, then the forecaster cannot be muthian at time $t$.

The optimization problem aims at searching for the agent who is the second best forecaster, according to the following definition.

Definition 1. Fix $t=1,2, \ldots$, and consider two agents $i_{1}, i_{2} \in\{1,2, \ldots, N\}$. Agent $i_{1}$ performs better than agent $i_{2}$ at time $t$ if and only if

$$
\left|L\left(x_{t}^{\left(i_{1}\right)}, \theta_{t}^{\left(i_{1}\right)}\right)-L\left(x_{t}^{\left(i_{1}\right)}, 0\right)\right|<\left|L\left(x_{t}^{\left(i_{2}\right)}, \theta_{t}^{\left(i_{2}\right)}\right)-L\left(x_{t}^{\left(i_{2}\right)}, 0\right)\right| .
$$

Definition 1 formalizes the fact that the second best forecaster is closer - w.r.t. the median - to the muthian agent of Remark 1.

To sum up, the selection of the second best forecaster is achieved through the solution of the following optimization problem:

$$
\inf _{\theta_{t}^{(i)} \in \mathbb{R}}\left|L\left(x_{t}^{(i)}, \theta_{t}^{(i)}\right)-L\left(x_{t}^{(i)}, 0\right)\right|, \quad \forall t \in \mathbb{N} .
$$

\section{Monte Carlo simulation results}

The optimization problem formalized in the previous Section is solved through extensive Monte Carlo simulations. As we shall see, the strategy is to implement a grid-search on a large number of $\theta$ 's. In this section we present the dataset and the definition of the variables 
employed for the simulation procedures. Results and the related discussion are contained in Sections 4 and 5.

In agreement with standard approaches (Elliott, et al. 2005; Capistran and Timmermann, 2009; Clements, 2009, 2014), we chose a multi-period forecasting horizon. This choice is also driven by the scope of enabling useful comparisons across our artificial agents through forward-looking decisions, still allowing potentially biased results. This aspect is relevant for our main aim. Thus, we consider $T \in \mathbb{N}$ such that $t=0,1,2, \ldots, T$, and set $T=19$, and $\delta=0.03$. We also let the initial datum of the GDP dynamics vary as $f_{0}=-0.025: 0.003:-0.001$. Moreover, it is assumed $f_{0}=\tilde{f}_{0}$, so that the initial error is $x_{0}=0$.

The selected value of the parameter $\rho$ comes out from a calibration on the basis of the quarterly growth rate of the real GDP for Italy. In particular, $\rho$ is equal to the 1992Q1-2014Q1 average of the above mentioned macroeconomic fundamental. Hence, we pose $\rho=0.0016$.

As already said, we pay specific attention to two bad economic environments: one moderately bad and a much more deteriorated one. To offer a more intuitive interpretation of the results, we also remove the dependence of $\alpha$ on time. Hence, we fix $\alpha_{t}=\alpha$, for each $t=1, \ldots, T$, and set $\alpha=\alpha_{d}, \alpha_{m}$, with $-1 \leq \alpha_{d}<<\alpha_{m}<0$. The parameters $\alpha_{d}, \alpha_{m}$ capture the cases of "deteriorated" and "moderately bad" cyclical phase, respectively. Assigning a value to the $\alpha$ 's is unnecessary. Indeed, the action of $\alpha$ impinges only on the diffusion coefficient $\sigma_{f}$ of the dynamics of $\left\{f_{t}\right\}_{t \geq 0}$ as written in equation (3). By following the hypotheses on $\sigma_{f}$, we set

$$
\sigma_{f}\left(f_{t}, \alpha_{d}\right)=0.4 \cdot f_{t}, \quad \sigma_{f}\left(f_{t}, \alpha_{m}\right)=0.01 \cdot f_{t} .
$$

The deterministic term of equation (3) is set to

$$
\mu_{f}\left(f_{t}\right)=\rho f_{t} .
$$

Function $\beta_{i}$ in (1) must be defined in order to let the deterministic trend of the real and of the forecasted GDP be the same in case of muthian agent. Hence, we select it as follows:

$$
\beta_{i}\left(\tilde{f}_{t}^{(i)}, \theta_{t}^{(i)}\right)=\frac{\rho}{1+\theta_{t}^{(i)}} \cdot \tilde{f}_{t}^{(i)} .
$$

To perform the grid search, we have generated a large number of synthetic agents. To this end, we have extracted $(J-3) \times T$ random numbers from an uniform distribution $U(-2,2)$. To be sure to include some cases of interest, we have added to such matrix three further rows with $T$ columns. The three rows are constant, and filled with $-2,1,2$, respectively. The resulting matrix $\theta$ has then dimension $J \times T$. The parameter $J$ has been set to 1000 . The procedure of generation of the matrix $\theta$ leads to the remarkable number of about ${ }^{2}$ $N=J^{T}$ synthetic agents.

\section{Results}

Simulations outcomes suggest interesting patterns of variation of the agents' predictive ability in variously deteriorated macroeconomic situations.

Tables 1-4 collect the obtained paths of $\theta$ in both cases of SB and median forecasters, for the cases of mild and severe crisis.

\footnotetext{
${ }^{2}$ The term "about" is here properly placed, because actually a negligible number of agents with some $\theta=-1$ and $\theta=0$ has been removed, to let functions $\beta$ 's in (7) be well-defined and to exclude the muthian case.
} 


\section{INSERT TABLE 1 ABOUT HERE}

Caption: Values of the $\theta$ 's for $t=1,2, \ldots, T$ and for $f_{0}=-0.025: 0.003:-0.001$. Case of mild crisis, second best agents.

INSERT TABLE 2 ABOUT HERE

Caption: Values of the $\theta$ 's for $t=1,2, \ldots, T$ and for $f_{0}=-0.025: 0.003:-0.001$. Case of mild crisis, median agents.

INSERT TABLE 3 ABOUT HERE

Caption: Values of the $\theta$ 's for $t=1,2, \ldots, T$ and for $f_{0}=-0.025: 0.003:-0.001$. Case of severe crisis, second best agents.

INSERT TABLE 4 ABOUT HERE

Caption: Values of the $\theta$ 's for $t=1,2, \ldots, T$ and for $f_{0}=-0.025: 0.003:-0.001$. Case of severe crisis, median agents.

Comparing the asymmetry parameters of our synthetic agents shows that SB agents can succeed in forecasting as good as muthians. As expected, however, this happens only occasionally and never happens for medians. Thus, our artificial economy is able to reflect the structural heterogeneity characterizing the real world where different types of agents inevitably coexist.

Another interesting finding is that the two groups under scrutiny are relatively more similar during mild economic periods than in more critical cyclical phases (see two illustrative examples in Figures 2 and 3). This outcome is consistent with the obvious fact that it is easier to forecast a constant than a volatile fundamental. Otherwise stated, economic stability is associated to macroeconomic variables with low volatility, which tends to limit the range of forecasting errors whatever the predictors' approach or skill. When the forecasting error is small, no matter whether agents predict optimally or median, the plausible range of the selected $\theta$ 's tend to show small oscillations around zero.

\section{INSERT FIGURE 2 ABOUT HERE}

Caption: Differences between SB and medians agent's $\theta$ 's in both types of crises. Case

$$
f_{0}=-0.025 \text {. }
$$

\section{INSERT FIGURE 3 ABOUT HERE}

Caption: Differences between SB and medians agent's $\theta$ 's in both types of crises. Case

$$
f_{0}=-0.001 \text {. }
$$

A third intriguing finding emerging from our simulations is that the less sophisticated forecasters' $\theta$ 's tend to be relatively smoother than the second best agents' ones. This holds regardless the size of the crisis (see Table 5).

\section{INSERT TABLE 5 ABOUT HERE}

Caption: Difference between the standard deviations w.r.t. $t$ of the $\theta$ 's for medians and SB forecasters. The cases of $f_{0}$ are distinguished.

Simulations highlight more details. The two groups of sub-optimal agents appear relatively more similar during mild economic stances, while they behave very differently amid dramatic economic turmoils. In particular, it seems that deep crises lead second best agents to show a sort of bang-bang behavior (see Tables 1 and 3). This also implies that, occasionally, 
naïve forecasters can outperform SB ones. We interpret this finding as follows. The lessschizoid behavior of medians mirrors the fact that - by construction - they are less attentive and have lower information and forecasting ability with respect to SB agents. Accordingly, medians are relatively less prone or prompt to seek and to include new information and, hence, to revise their beliefs. One is then tempted to say that median forecasters follow a more "conservative behavior" than SB forecasters who, it is worth recalling, seek to mimic as much as possible the Muth-optimal agents' behavior. In doing that great recessions induce SB agents to commit larger errors. The reason for this is twofold. First, economic turbulence tends to enlarge muthians' forecasting errors. In turn, this induces greater biases in SB forecasters. Second, for reasons such as greater mass media coverage, major crises broaden the available information set. Thus, unlike the inattentive medians, SB agents revise their forecasts because they quickly exploit any new information. The problem with the SB promptness is that, as emphasized by Doms and Morin (2004), the media tends to emphasize bad economic news. Consequently, as argued by Blendon et al. (1997), the public's biases can be attributed to the media's focus on bad news (see also Bovi, 2009). On this topic, many years ago Simon (1971) noted that a wealth of information creates a poverty of attention. Summarizing, the simple availability of more information neither necessarily mean better information nor better forecasting performances. All in all, our simulations suggest a rewording of Ackoff's famous phrase: it is not silly to not look for an optimal solution to a mess (Ackoff, 1977).

\section{Concluding Remarks}

This paper has dealt with the effects of bad economic periods on the expectation formation process (EFP) of different kinds of forecasters, characterized by heterogeneous predictive ability. The basic idea is that expectations are a key factor in economics and that people behave differently. Just to mention, there are quite significant and well-known incentives to become a sport champion or to win a Nobel Prize, yet very few persons succeed in the endeavor. The brutal truth is that the majority lags behind or gives up. By the same token lay forecasters may learn, but it is unrealistic to think that all of them - even in the long run - will achieve Muth-optimal and, hence, homogeneous forecasts. Studying inflation expectations, some authors (Ranyard et al. 2008, Armantier et al. 2013) have found that lay individuals tend to form more heterogeneous predictions than those of professional forecasters. Other authors have then argued that individuals use information derived from their personal experience as consumers, which can be both diverse and inaccurate (Bruine de Bruin et al. 2011, Madeira and Zafar, 2013). The foregoing leads to confirm that people's performances are structurally different. The situation is made even more complex - and more interesting to study - when the fundamental to predict, the real GDP growth rate, is well below zero and highly volatile.

This paper may be though of as complementing two strands of the literature. First, according to the rational expectations hypothesis all agents use the "true" model and homogeneous expectations naturally arise. This said, the new evidence on the limitations of the rational expectations hypothesis has led researchers to reexamine the process of expectation formation and its implications for macroeconomic models. The adaptive learning approach (Evans and Honkapohja, 2001) assumes that agents are boundedly rational, but as smart as econometricians. In our setting, econometricians may be easily classified as SB forecaster. Accordingly agents - who know the correct model of the economy but do not know the pa- 
rameters - in order to produce forecasts infer parameters as any econometrician would do - by relentlessly estimating the model. Bounded rationality has also been imposed in other forms and one may say that three main sources of heterogeneity have been documented: agents produce heterogeneous forecasts because $i$ ) they are using different models, $i i$ ) they have different information sets, and iii) they have differing levels of capacity to process information. Typically, these approaches assume that - at least in the long-run - differences among agents should diminish, eventually resulting in identical forecasts. In this respect, our model and findings suggest a different perspective. In particular, we model structural heterogeneity taking advantage of three approaches elaborated to study the EFP, namely, Muth-rational expectations, adaptive learning, and heuristics and biases.

Second, this paper contributes to the literature by examining the effects of macroeconomic dynamics on the EFP via asymmetric loss functions. Somewhat in line with our results, a number of authors (Elliott, et al. 2005; Capistran and Timmermann, 2009; Clements, 2009, 2014) has shown that disagreement in expectations varies systematically over time in a way that reflects the level and variance of the macroeconomic fundamental under scrutiny. Side-by-side with the use of a macroeconomic fundamental and of the LINEX, we have taken advantage of this literature even in our choice to examine a $T$-step ahead forecast horizon. On the other hand, $T$-step ahead forecasts are very common both in macroeconomic practice and in econometric analysis. We depart from this literature in having adopted methodological tools grounded in the operational research theory. Specifically, we have developed a dynamic stochastic optimization problem.

Monte Carlo simulations have shown intriguing patterns, shedding some light on how the asymmetry parameters - i.e. the agents' predictive ability - vary according to how serious the crisis is. The less sophisticated forecasters in our setting - i.e.: the medians - never perform as muthians and SB agents do that only occasionally. This regardless of the size of the crisis. This aspect correctly reflects the real world. Differently from the above mentioned literature, in our artificial economy heterogeneity is thus a structural trait. Even more intriguingly, simulations also show that the behavior of medians tends to be relatively smoother than that of SB agents, and that the difference between them during major crises. In particular, great recessions lead SB agents' predictions to be relatively more biased. An explanation is that dramatic crises broaden the available information set (e.g., due to greater mass media coverage), leading second-best agents, who are more attentive than medians, to revise their forecasts. The problem is that more information does not necessarily lead to better forecasting performances. In general, as stressed by Simon (1971) a wealth of information creates a poverty of attention. The problem is compounded by the fact that, as suggested by the literature on rational inattention (Reis, 2006, 2009), decision-makers have a limited amount of attention available and have to decide how to allocate it. This limitation may lead to a rational inattention that is somewhat in line with our outcomes. All considered, this paper supports a rewording of Ackoff's famous phrase: it is not silly to not look for an optimal solution to a mess.

\section{References}

[1] Ackoff, R.L., 1977. Optimization + objectivity = optout. European Journal of Operational Research 1(1), 1-7.

[2] Aizenman, J., Pinto, B., (eds.) Managing Economic Volatility and Crises: A Practitioner's Guide. Cambridge: Cambridge University Press, 2005. 
[3] Armantier, O.,Bruine de Bruin, W., Potter, G. Topa, G., van der Klaauw, W., Zafar, B. 2013. Measuring Inflation Expectations. Annual Review of Economics 5, 273-301.

[4] Bardi, M., Capuzzo-Dolcetta, I., Optimal control and viscosity solutions of HamiltonJacobi-Bellman equation. Birkhäuser Boston, Inc., Boston, MA; 1997.

[5] Barone, R., Cerqueti, R., Quaranta, A.G., 2012. Illegal financier and usurer behavior. European Journal of Law and Economics 34(2), 265-277.

[6] Bayram, A., Solaka, S., Johnson, M., 2014. Stochastic models for strategic resource allocation in nonprofit foreclosed housing acquisitions. European Journal of Operational Research 233(1), 246-262

[7] Blendon, R., Benson, J., Brodie, M., Morin, R., Altman, D., Gitterman, D., Brossard, M., James, M., 1997. Bridging the gap between the publics and economists views of the economy. Journal of Economic Perspectives 11, 105-188.

[8] Blueschke, D., Blueschke-Nikolaeva, V., Savin, I., 2013. New insights into optimal control of nonlinear dynamic econometric models: Application of a heuristic approach. Journal of Economic Dynamics and Control 37(4), 821-837.

[9] Borkar, V.S., Optimal control of diffusion processes. Pitman Research Notes in Mathematics Series, Longman Scientific \& Technical: Harlow; 1989.

[10] Bovi, M., 2013. Are the representative agent's beliefs based on efficient econometric models?. Journal of Economic Dynamics and Control 37(3), 633-648.

[11] Bovi, M., 2009. Economic versus Psychological Forecasting. Evidence from Consumer Confidence Surveys. Journal of Economic Psychology 30(4), 563-574.

[12] Bruine de Bruin, W., van der Klaauw, W., Topa, G., 2011. Expectations of inflation: The biasing effect of thoughts about specific prices. Journal of Economic Psychology $32(5), 834-845$.

[13] Cao, Y., Hussaini, M.Y., Zang, T.A., 2003. An Efficient Monte Carlo Method for Optimal Control Problems with Uncertainty. Computational Optimization and Applications 26, 219-230.

[14] Capistran, C., Timmermann, A., 2009. Disagreement and Biases in Inflation Expectations. Journal of Money, Credit and Banking 41(2-3), 365-396.

[15] Castellano, R., Cerqueti, R., 2014. Mean-variance portfolio selection in presence of unfrequently traded stocks. European Journal of Operational Research 234(2), 442449 .

[16] Castellano, R., Cerqueti, R., 2012. Optimal consumption/investment problem with light stocks: A mixed continuous-discrete time Approach. Applied Mathematics and Computation 218(12), 6887-6898.

[17] Cerqueti, R., Quaranta, A.G., 2012. The Perspective of a Bank in Granting Credits: an Optimization Model. Optimization Letters 6(5), 867-882. 
[18] Chiarella, C., Khomin, P., 1999. Adaptively evolving expectations in models of monetary dynamics: The fundamentalists forward looking. Annals of Operations Research $89,21-34$.

[19] Chiarella, C., He, T., 2001. Asset Price and Wealth Dynamics under Heterogeneous Expetations. Quantitative Finance 1, 509-526.

[20] Consiglio, A., Russino, A., 2007. How does learning affect market liquidity? A simulation analysis of a double-auction financial market with portfolio traders. Journal of Economics Dynamics and Control 31(6), 1910-1937.

[21] Consiglio, A., Staino, A., 2012. A stochastic programming model for the optimal issuance of government bonds. Annals of Operations Research 193(1), 159-172.

[22] Dietz, S., 2012. A Nonlinear Model of Economic Data Related to the German Automobile Industry. International Journal of Applied Operational Research 2(1), 23-33.

[23] Doms, M., Morin, N., 2004. Consumer sentiment, the economy, and the news media, finance and economics discussion series (Vol. 51). Washington, DC: Divisions of Research \& Statistics and Monetary Affairs Federal Reserve Board.

[24] European Commission, 2007. The Joint Harmonised EU Programme of Business and Consumer Surveys, User Guide, European Commission. Directorate- General for Economic and Financial Affairs.

[25] Fleming, W.H., Soner, H.M., Controlled Markov processes and viscosity solutions. Applications of Mathematics (New York), Springer-Verlag: New York; 1993.

[26] Kahneman, D., Tversky, A., 1974. Judgment under uncertainty: Heuristics and biases. Science 185, 11241131.

[27] Kirby, M.W., 2007. Paradigm Change in Operations Research: Thirty Years of Debate. Operations Research 55(1), 1-13.

[28] Krylov, N.V., Controlled diffusion processes. Springer-Verlag: New York; 1980.

[29] Madeira, C., Zafar, B., 2014. Heterogeneous Inflation Expectations and Learning. Federal Reserve Bank of New York Staff Reports, n. 536.

[30] McAllister, P.H., 1991. Adaptive approaches to stochastic programming. Annals of Operations Research 30(1), 45-62.

[31] Muth, J.F., 1961. Rational Expectations and the Theory of Price Movements. Econometrica 29(3), 315-335.

[32] Niemira, M.P., Saaty, T.L., 2004. An analytic network process model for financial-crisis forecasting. International Journal of Forecasting 20(4), 573-587.

[33] Pesaran, M.H., Weale, M., 2006. Survey expectations. In: G. Elliott, C. Granger, A. Timmermann (Eds.), Handbook of economic forecasting. Amsterdam: North Holland.

[34] Ranyard, R., Missier, F.D., Bonini, N., Duxbury, D., Summers, B., 2008. Perceptions and expectations of price changes and inflation: A review and conceptual framework. Journal of Economic Psychology 29(4), 378-400. 
[35] Rios-Rull, J.-V., 2001. Computation of Equilibria in Heterogeneous Agent Models. In: Marimon, R. and A. Scott, eds., Computational Methods for the Study of Dynamic Economies: An Introduction, Oxford University Press, 238-280.

[36] Sevim, C., Oztekinb, A., Balic, O., Gumusd, S., Guresenc, E., 2014. Developing an early warning system to predict currency crises. European Journal of Operational Research 237(3), 1095-1104.

[37] Simon, H.A., 1971. Designing Organizations for an Information-Rich World. In: Martin Greenberger, Computers, Communication, and the Public Interest, Baltimore. MD: The Johns Hopkins Press. pp. 40-41.

[38] Engels, J.R., 1992. On simulation and optimization of macroeconometric models. European Journal of Operational Research 61(3), 357-369.

[39] Woodford, M., 2009. Convergence in macroeconomics: Elements of the new synthesis. American Economic Journal: Macroeconomics 1(1), 267-279.

[40] Yong, J., Zhou, X.Y., Stochastic controls. Hamiltonian systems and HJB equations. Applications of Mathematics, Springer-Verlag: New York; 1999.

\section{Appendix}

We present the numerical procedures in a block-wise form.

First block

The first building block of consists of the generation of the dynamics of agents' GDP forecast $\left\{\tilde{\mathbf{f}}_{t}\right\}_{t}$ in (1).

(A.1) set $j=1$;

(A.2) $\operatorname{set} t=1$;

(A.3) set $\tilde{f}_{j, t}=\tilde{f}_{0}$;

(A.4) set $t=t+1$ and go to step (A.5);

(A.5) if $t=T+1$, go to step (A.7). Otherwise, go to step (A.6);

(A.6) set $\tilde{f}_{j, t}=\tilde{f}_{j, t-1}+\rho /(1+\theta(j, t)) * \tilde{f}_{j, t-1}$ and go to step (A.4);

(A.7) set $j=j+1$ and go to step (A.8);

(A.8) if $j=J+1$, stop. Otherwise, go to step (A.2).

Indices $t=1, \ldots, T$ and $j=1, \ldots, J$ represent time and the components of the vectors $\theta_{t}$, for each $t$.

\section{Second block}

In the second building block of the application, the stochastic dynamics of the true GDP $\left\{f_{t}\right\}_{t}$ is constructed. In particular, $H=1,000$ scenarios are simulated by extracting $H=$ 1,000 times the standard normal variable $\epsilon$ in equation (3). The values of $\alpha$ are also considered. 
(B.1) set $a=1$;

(B.2) set $h=1$;

(B.3) set $t=1$;

(B.4) set $f_{t, h, a}=f_{0}$;

(B.5) set $t=t+1$ and go to step (B.6);

(B.6) if $t=T+1$, go to step (B.8). Otherwise, go to step (B.7);

(B.7) set $f_{t, h, a}=f_{t-1, h, a}+\rho * f_{t-1, h, a}+\sigma(a) * f_{t-1, h, a} * N_{h}(0,1)$ and go to step (B.5);

(B.8) set $h=h+1$ and go to step (B.9);

(B.9) if $h=H+1$, go to step (B.10). Otherwise, go to step (B.3);

(B.10) set $a=a+1$ and go to step (B.11);

(B.11) if $a=3$, stop. Otherwise, go to step (B.2).

Indices $h=1, \ldots, H$ and $a=1,2$ point to the random scenarios and to the intensity of the crisis, respectively. In particular, $a=1,2$ stand for $\alpha=\alpha_{m}, \alpha_{d}$, respectively.

In accord to the setting of the problem, function $\sigma(a)$ introduced in step (B.7) is such that $\sigma(1)=0.01, \sigma(2)=0.4$.

$N_{h}(0,1)$ is the adopted notation for the standard normal variable, which is extracted in correspondence of the values of index $h$.

\section{Third block}

The third block aims at constructing the dynamics of the errors $\left\{x_{t}\right\}_{t}$ as in formula (4).

(C.1) set $j=1$;

(C.2) set $a=1$;

(C.3) set $h=1$;

(C.4) set $t=1$;

(C.5) set $x_{j, t, h, a}=0$;

(C.6) set $t=t+1$ and go to step (C.7);

(C.7) if $t=T+1$, go to step (C.9). Otherwise, go to step (C.8);

(C.8) set $x_{j, t, h, a}=f_{t, h, a}-\tilde{f}(j, t)$ and go to step (C.6);

(C.9) set $h=h+1$ and go to step (C.10);

(C.10) if $h=H+1$, go to step (C.11). Otherwise, go to step (C.4);

(C.11) set $a=a+1$ and go to step (C.12);

(C.12) if $a=3$, go to step (C.13). Otherwise, go to step (C.3); 
(C.13) set $j=j+1$ and go to step (C.14);

(C.14) if $j=J+1$, stop. Otherwise, go to step (C.2).

By using the definition of the loss function $L$ in (5) and the quantities $\theta(j, t)$ and $x_{j, t, h, a}$ introduced in the previous steps of the numerical procedure, we have obtained a $J \times T \times H \times 2$ matrix $\tilde{\mathbf{L}}=\left(\tilde{L}_{j, t, h, a}\right)_{j, t, h, a}$ as follows:

$$
L_{j, t, h, a}=\left|\frac{1}{\theta(j, t)^{2}} *\left[\exp \left((\theta(j, t)) * x_{j, t, h, a}\right)-\theta(j, t) * x_{j, t, h, a}-1\right]-\left(x_{j, t, h, a}\right)^{2}\right| .
$$

Recalling that the index $h$ is associated to the simulated scenarios, we have then taken the average on $h$ and obtain the matrix $\mathbf{L}=\left(L_{j, t, a}\right)_{j, t, a}$ :

$$
L_{j, t, a}=\frac{1}{H} \sum_{h=1}^{H} \tilde{L}_{j, t, h, a} .
$$

The cases $a=1$ and $a=2$ have been distinguished. Fixed $a=\bar{a}$, the median and the second best forecaster have been identified as follows:

- Median forecaster

For each $\bar{t}=1,2, \ldots, T$, we have taken the median of $\left(L_{j, \bar{t}, \bar{a}}\right)_{j}$, say for $j=j_{\bar{t}}^{\star}$, and identified the corresponding $\theta\left(j_{\bar{t}}^{\star}, \bar{t}\right)$. When the number of the data is pair, the median do not correspond to any $\theta$. In this case, we have sorted $\left(L_{j, \bar{t}, \bar{a}}\right)_{j}$ in increasing order and found the index $\bar{j}_{\bar{t}}$ such that the median of $\left(L_{j, \bar{t}, \bar{a}}\right)_{j}$ is $1 / 2\left(L_{\bar{j}_{\bar{t}}-1, \bar{t}, \bar{a}}+L_{\bar{j}_{\bar{t}}, \bar{t}, \bar{a}}\right)$. We have then set $j_{\bar{t}}^{\star}=\bar{j}_{\bar{t}}$, hence leading to $\theta\left(j_{\bar{t}}^{\star}, \bar{t}\right)=\theta\left(\bar{j}_{\bar{t}}, \bar{t}\right)$.

The trajectory of the $\theta$ related to the median forecaster is

$$
\theta_{\text {median }}=\left(\theta\left(j_{1}^{\star}, 1\right), \theta\left(j_{2}^{\star}, 2\right), \ldots, \theta\left(j_{T}^{\star}, T\right)\right) .
$$

- Second best forecaster

For each $\bar{t}=1,2, \ldots, T$, we have taken the minimum over $j$ of $\left(L_{j, \bar{t}, \bar{a}}\right)_{j}$, which is assumed to be attained for $j=j_{\bar{t}}^{\bullet}$, and identified the corresponding $\theta\left(j_{\bar{t}}^{\bullet}, \bar{t}\right)$.

The trajectory of the $\theta$ related to the second best forecaster is

$$
\theta_{s b}=\left(\theta\left(j_{1}^{\bullet}, 1\right), \theta\left(j_{2}^{\bullet}, 2\right), \ldots, \theta\left(j_{T}^{\bullet}, T\right)\right) .
$$

